\title{
Psicooncología
}

ISSN: $1696-7240$

\section{Ansiedad, depresión, percepción y sentimientos de mujeres con cáncer indicadas para cirugía de exenteración pélvica}

\author{
Sílvia Abduch Haas ${ }^{1, *}$; Daniela Centenaro Levandowski²; Antônio Nocchi Kalil ${ }^{3}$
}

Recibido: 1 de junio de 2017 / Aceptado: 25 de agosto de 2017

Resumen. Objetivo: el presente estudio evalúa señales de depresión y ansiedad en mujeres con cáncer indicadas para cirugía de exenteración pélvica (EP), e identifica sus percepciones y sentimientos ante la EP. Método: Participaron en el estudio seis mujeres (37 a 64 años) que recibían tratamiento oncológico en un hospital del sur de Brasil. Las pacientes rellenaron una ficha de datos sociodemográficos y respondieron a la Escala Hospitalaria de Ansiedad y Depresión (HADS). También fueron realizadas entrevistas semiestructuradas. Resultados: En general, los resultados revelan reacciones emocionales a la indicación de EP para las participantes. Se verificó la presencia de ansiedad y depresión (50\% y $66,7 \%$, respectivamente) en el periodo preoperatorio. A partir del análisis de las entrevistas fue posible identificar dudas y temores sobre el resultado de la cirugía, así como sobre las expectativas de cura del cáncer. También aparecieron sentimientos de miedo, preocupación y ansiedad ante las ostomías. Conclusión: Estudios longitudinales pueden ampliar la comprensión del impacto de la EP, considerando también el periodo postoperatorio. Se subraya la necesidad de un seguimiento psicológico preoperatorio para mujeres indicadas para este procedimiento altamente especializado e invasivo.

Palabras clave: Exenteración pélvica; aspectos psicológicos; depresión; ansiedad; cáncer.

\section{[en] Anxiety, depression, perceptions and feelings of women with cancer referred for pelvic exenteration surgery}

\footnotetext{
Abstract. Objective: This study aimed to evaluate depression and anxiety in women with cancer who were referred for pelvic exenteration (PE), and to identify their perceptions and feelings regarding PE. Methods: Six women (aged between 37 and 64 years,) undergoing cancer treatment at a hospital in the city of Porto Alegre, Brazil, took part in the study. Sociodemographic data were collected and a semi-structured interview and the Hospital Anxiety and Depression Scale (HADS) were employed. Results: The overall findings revealed emotional implications related to PE. Anxiety and depression were experienced by the participants ( $50 \%$ and $66.7 \%$, respectively) before the surgery. The interviews showed doubts and fears regarding the surgical outcomes as well as expectations for cancer cure.

1 Hospital Santa Rita - Irmandade Santa Casa de Misericórdia de Porto Alegre, Brasil.

E-mail: silvia.haas@santacasa.tche.br

2 Departamento de Psicología. Programa de Pós-Graduação em Ciências da Saúde. Universidade Federal de Ciências da Saúde de Porto Alegre, Brasil.

E-mail: danielal@ufcspa.edu.br

3 Universidade Federal de Ciências da Saúde de Porto Alegre. Hospital Santa Rita - Irmandade Santa Casa de Misericórdia de Porto Alegre, Brasil.

E-mail: ankalil@terra.com.

* Dirección de correspondencia: Sílvia Abduch Haas. Av. Taquara, 596/201, Bairro Petrópolis, CEP 90460210. Porto Alegre, RS, Brasil. E-mail: silvia.haas@santacasa.tche.br
} 
Anxiety, fear and worries associated with ostomies also emerged. Conclusion: Longitudinal studies may contribute to a broader understanding of the impact of this surgery, including the postoperative period. It is possible to highlight the need to psychological assistance before the surgery to women referred to this highly specialized and invasive medical procedure.

Keywords: Pelvic exenteration; psychological aspects; depression; anxiety; cancer.

Sumario. 1. Introducción 2. Método 3. Análisis de datos 4. Resultados 5. Discusión 6. Conclusiones 7. Agradecimientos 8. Referencias bibliográficas.

Cómo citar: Abduch Haas S, Centenaro Levandowski D, Nocchi Kalil A. Ansiedad, depresión, percepciones y sentimientos de mujeres con cáncer indicadas para cirugía de exenteración pélvica. Psicooncología 2017;14:279-294. Doi: 10.5209/PSIC.57086.

\section{Introducción}

El cáncer de cuello de útero es el cuarto tumor más frecuente como causa de óbito entre la población femenina brasileña. Estimaciones del Instituto Nacional del Cáncer ${ }^{(1)}$ apuntan que, durante el año 2016, más de 16.000 mujeres brasileñas serían diagnosticadas con este tipo de tumor.

Cuando el diagnóstico es precoz, existen grandes oportunidades de cura. Actualmente, el $44 \%$ de los casos diagnosticados son de lesión precursora del cáncer, llamada in situ $^{(1)}$, que puede ser prevenida si es tratada precozmente o identificada por el examen citopatológico (Papanicolaou). Sin embargo, la prevención anual no siempre es parte de la rutina de la población nacional: en Brasil, cerca del $30 \%$ de las mujeres nunca se hicieron un examen citológico. La enfermedad es diagnosticada en fase avanzada en un $70 \%$ de los $\operatorname{casos}^{(2)}$ y menos del $15 \%$ de la población femenina brasileña se involucra en programas de prevención del cáncer de cuello de útero, lo que ayuda a explicar la alta incidencia de la patología ${ }^{(3)}$. Aunque los métodos de análisis sean seguros, sensibles y disponibles para la población, se estima que el $40 \%$ de las brasileñas nunca lo ha realizado, y solo descubren la enfermedad precozmente al utilizar los servicios públicos de obstetricia para tener hijos. En caso contrario, detectan la enfermedad en fase avanzada después de los 35 años $^{(4)}$.

Para las mujeres que desarrollan tumores malignos pélvicos recurrentes o tumores primarios avanzados, así como otros tipos de cáncer ginecológico, de recto y urológico, puede ser indicada la realización de la cirugía de Exenteración Pélvica ${ }^{(5)}$. La Exenteración Pélvica (EP) es una técnica quirúrgica radical, que incluye la remoción de órganos pélvicos (por ejemplo, vagina, vulva, perineo, vejiga, útero, ano y uretra) y generalmente implica el uso de bolsas colectoras (urostomía y/o colostomía) ${ }^{(6-9)}$. Este procedimiento requiere un largo periodo de hospitalización y cuidados especiales ${ }^{(10)}$.

La EP se emplea cuando las pacientes son contraindicadas para nuevos ciclos de radioterapia y quimioterapia, siendo considerada una segunda oportunidad de vida (10). Así, la indicación para este procedimiento se da para el $78 \%$ de las pacientes con los tipos de cáncer citados ${ }^{(2)}$. Aun así, en gran parte de los casos, se trata de una cirugía de carácter paliativo, ya que los índices de supervivencia tras la EP se sitúan entre el 23 y el $61 \%^{(2,10)}$. Los índices de mortalidad son de aproximadamente el $23 \%$ en el postoperatorio ${ }^{(11)}$. 
Por todos estos aspectos, es necesaria una evaluación detallada e interdisciplinar de la paciente y su familia, que considere el tiempo de supervivencia versus la inversión física y emocional para la realización de la EP. La posibilidad de óbito debe ser considerada y discutida, al igual que las alteraciones y cambios corporales consecuentes de la intervención, incluyendo el uso de bolsas colectoras ${ }^{(12)}$, para que la paciente esté informada de las posibilidades y limitaciones de la cirugía. Sin embargo, este no siempre es el panorama encontrado, como se constata en el estudio cualitativo realizado por Wright et al. ${ }^{(13)}$ con 10 pacientes canadienses con cáncer colorrectal que habían realizado la EP. Según los autores, las pacientes revelaron la percepción de haber recibido informaciones incompletas del equipo sobre su enfermedad, el tratamiento y sobre la posibilidad de óbito. Por tanto, es importante la disponibilidad del equipo para mantener una comunicación clara con las pacientes, sus compañeros y demás familiares sobre los efectos colaterales y secuelas de la EP.

La experiencia de tener cáncer de cuello uterino, así como los tratamientos indicados (quimioterapia, radioterapia y braquiterapia), conllevan un impacto emocional importante para las mujeres, no siendo raro el surgimiento de sentimientos de culpa y la realización de una retrospectiva de sus vidas, como tentativa de comprender el surgimiento de la enfermedad ${ }^{(14)}$. Ante el diagnóstico de cáncer, el individuo vive un momento estresante y perturbador, pudiendo presentar ansiedad, depresión, delirium, ideación suicida o incluso llevar a cabo el suicidio. La enfermedad y los efectos de los tratamientos generan cambios muy significativos en la vida de las mujeres, pudiendo comprometer su calidad de vida y bienestar ${ }^{(15,16)}$. Ansiedad y depresión son las alteraciones emocionales más frecuentes en estas pacientes, presentando altos índices en las internaciones hospitalarias ${ }^{(17,18)}$. Especialmente, en el caso de las mujeres, se verifica que aquellas que se encuentran deprimidas necesitan tiempo, auxilio psicológico y apoyo familiar para la elaboración del dolor por la amputación de partes del cuerpo que son símbolos de feminidad, como el útero, la vagina y los ovarios ${ }^{(19)}$, así como para asimilar la imposibilidad gestacional ${ }^{(20)}$.

Desde esta perspectiva, Hawighrst-Knapstein et al. ${ }^{(21)}$, al evaluar la Calidad de Vida $(\mathrm{CV})$ en pacientes americanas con cáncer genital, que habían realizado la EP, observaron que los cambios en su cuerpo, en la imagen corporal y en la sexualidad pasaron a ser foco de incertidumbre y sufrimiento, acarreando perjuicios psicosociales. Además, notaron que la frustración y la pérdida de CV eran mayores en los casos en que las mujeres fueron contraindicadas para la reconstrucción vaginal. Otros estudios también han abordado la $\mathrm{CV}$ con este público y considerado la necesidad de una atención especial a las pacientes, debido al impacto negativo de la $\mathrm{EP}^{(10,22-24)}$.

Además, es destacable que el luto generado por los cambios corporales puede desencadenar cuadros depresivos reactivos en estas mujeres, no siendo rara la evolución para un trastorno depresivo mayor, caso haya comorbilidades asociadas (25). Así, conocer y comprender los aspectos psicológicos de estas pacientes es esencial para los equipos de salud.

A pesar de la importancia del tema, en la literatura brasileña aun son escasos los estudios sobre los aspectos emocionales de mujeres orientadas para la cirugía de EP. La baja incidencia de realización de este tipo de procedimiento superespecializado en el país puede explicar en parte esta laguna. Aun así, verificar empíricamente 
los niveles de ansiedad y depresión de estas mujeres, la forma en que perciben su cuerpo y su relación conyugal, y la presencia o no de una red de apoyo, entre otras cuestiones, puede ser una importante herramienta para la cualificación de la atención psicológica prestada a ellas, incluso en la preparación para la cirugía. El presente estudio buscó evaluar señales de depresión y ansiedad en mujeres con cáncer indicadas para la cirugía de EP e identificar sus percepciones y sentimientos ante la EP.

\section{Método}

\subsection{Participantes}

Seis mujeres, con edades entre 37 y 64 años (media=48,8 años; DT=9,9), que recibían tratamiento oncológico en el Hospital Santa Rita (HSR - ISCMPA), centro de referencia para el tratamiento del cáncer en el sur de Brasil. Las pacientes fueron remitidas por los equipos de cirugía oncológica y ginecológica al Servicio de Psicología (SP) del HSR tras haber recibido indicación para la EP.

La colecta de datos se realizó entre agosto de 2013 y junio de 2014. Se incluyó en el estudio a todas las pacientes indicadas para la cirugía de EP en este periodo. De las nueve pacientes invitadas a colaborar con la investigación, dos fueron contraindicadas durante la colecta, debido a la posibilidad de realización de quimioterapia, y otra candidata falleció. Así, seis mujeres integraron el presente estudio. La tabla 1 describe de forma detallada las principales características (sociodemográficas y de salud) de las participantes.

Tabla 1. Caracterización de las participantes.

\begin{tabular}{lc}
\hline Características & $\mathbf{n}=\mathbf{6}$ \\
\hline Edad (años) - media \pm DT & $48,8 \pm 9,9$ \\
Tiempo de diagnóstico (años) - media \pm DT & $2,4 \pm 1,1$ \\
Estado civil - n (\%) & \\
$\quad$ Casada/Unión estable & $4(66,7)$ \\
$\quad$ Separada & $1(16,7)$ \\
$\quad$ Viuda & $1(16,7)$ \\
Número de hijos - md (mín - máx) & $3(1-6)$ \\
Quimioterapia - n (\%) & $4(66,7)$ \\
Radioterapia - n (\%) & $5(83,3)$ \\
Braquiterapia - n (\%) & $3(50,0)$ \\
Cirugía - n (\%) & $1(16,7)$ \\
Realizó preventivo - n (\%) & $5(83,3)$ \\
Diagnóstico - n (\%) & \\
$\quad$ CA cuello del útero & $5(83,3)$ \\
$\quad$ CA epidermoide de canal anal & $1(16,7)$ \\
\hline CA Cáncer
\end{tabular}

$\mathrm{CA}=$ Cáncer. 


\subsection{Delineación, procedimientos e instrumentos}

Se trata de un estudio de carácter transversal y delineación mixta ${ }^{(26)}$, en el cual fueron empleados instrumentos cualitativos y cuantitativos para la colecta de datos.

A partir de la orientación de las pacientes por los equipos de cirugía y ginecología del HSR al SP, se realizó la invitación para participar en el estudio, con la presentación de la investigación y el formulario de Consentimiento Informado. En caso de aceptación, se inició la colecta de datos con la realización de una entrevista semiestructurada para examinar las vivencias de las pacientes en el periodo preoperatorio, incluyendo la reacción ante el diagnóstico y manejo del cáncer, las alteraciones provocadas por la enfermedad, la presencia de una red de apoyo, el proceso de decisión ante la EP y los planes futuros y expectativas tras la cirugía, entre otros temas. Esas entrevistas fueron grabadas en audio para posterior transcripción.

Después, fueron colectados datos sociodemográficos y de salud de las pacientes mediante una ficha elaborada para el presente estudio. También era aplicada la Escala Hospitalaria de Ansiedad y Depresión (HADS), que identifica síntomas de ansiedad y depresión en pacientes de hospitales clínicos no psiquiátricos. Esta escala está compuesta por 14 preguntas de opción múltiple, que generan dos subescalas (ansiedad y depresión) de siete elementos cada una. La puntuación global de cada subescala varía entre 0 y 21 , y la puntuación igual o superior a 9 indica la presencia de depresión y/o ansiedad ${ }^{(27)}$. Esta escala fue validada para Brasil por Botega et al. ${ }^{(28)}$ con alphas de Cronbach de 0,68 (ansiedad) y 0,77 (depresión).

La duración media de la colecta de datos fue de 60 minutos. Antes de la colecta de datos propiamente dicha, se realizó un estudio piloto para verificar la adecuación del guión de la entrevista a los objetivos del estudio.

\section{Análisis de datos}

La HADS fue analizada conforme las instrucciones de los autores ${ }^{(27,28)}$. El resultado, así como los demás datos cuantitativos (sociodemográficos y de salud), fueron registrados en el programa Statistical Package for the Social Sciences (SPSS) versión 17.0. Fue realizado un análisis estadístico descriptivo, en el cual las variables cuantitativas fueron descritas por media y desviación típica o mediana y rango, mientras que las variables categóricas fueron descritas por frecuencias absolutas y relativas. Para evaluar una eventual asociación entre variables cuantitativas (HADS, edad y tiempo de diagnóstico), se aplicó el test de correlación de Spearman. El nivel de significación adoptado fue de $5 \%(\mathrm{p} \leq 0,05)$.

Se realizó un análisis cualitativo de las entrevistas según la propuesta de Laville y Dionne $^{(29)}$. Fue adoptado un modelo mixto de análisis, en el cual algunas categorías fueron inicialmente postuladas en base a los conocimientos previos de los autores y con las preguntas del guión de la entrevista, mientras que otras categorías surgieron tras sucesivas lecturas del material. Con la categorización final, se realizó la interpretación de los datos ${ }^{(29)}$. 


\subsection{Aspectos éticos}

El estudio fue aprobado por el Comité de Ética en Investigación de la Universidade Federal de Ciências da Saúde de Porto Alegre (UFCSPA) y de la Irmandade Santa Casa de Misericórdia de Porto Alegre (ISCMPA), por el protocolo 367.054, de 21/08/13. Además de las precauciones éticas ya mencionadas, resaltamos que fue proporcionado a las participantes el mismo tratamiento normalmente ofrecido por el SP a los pacientes oncológicos internos en el HSR. Además, al constatarse la necesidad, fue indicada psicoterapia individual ambulatoria en la misma institución para dos participantes.

\section{Resultados}

\subsection{Análisis Cuantitativo}

En lo relativo a la HADS, de las seis participantes, $66,7 \%(\mathrm{n}=4)$ presentaron depresión y $50 \%(n=3)$ ansiedad en el periodo preoperatorio, como puede apreciarse en la Tabla 2. Solo una participante no presentó ninguno de estos síntomas.

Tabla 2. Resultados de la Escala Hospitalaria de Ansiedad y Depresión (HADS).

\begin{tabular}{lc}
\hline HADS & $\mathbf{n}=\mathbf{6}$ \\
\hline Ansiedad & \\
Puntuación Total - Media (mín - máx) & $9(2-16)$ \\
Clasificación - n (\%) & \\
$\quad$ Con & $3(50,0)$ \\
$\quad$ Sin & $3(50,0)$ \\
Depresión & \\
Puntuación Total - Media (mín - máx) & $9,5(1-17)$ \\
Clasificación - n (\%) & \\
$\quad$ Con & $4(66,7)$ \\
$\quad$ Sin & $2(33,3)$ \\
\hline
\end{tabular}

\subsection{Análisis Cualitativo}

A partir del análisis de contenido cualitativo de las entrevistas, tres categorías temáticas emergieron: Reacciones ante la indicación quirúrgica, Sentimientos en relación a las ostomías, y Sentimientos en relación a las repercusiones de la EP en el cuerpo y la sexualidad. A seguir constan las categorías, sus respectivas subcategorías y fragmentos de las entrevistas ilustrativas de las mismas. 


\section{1) Reacciones ante la indicación quirúrgica}

En esta categoría fueron englobados los fragmentos de las entrevistas en los que las participantes refirieron reacciones ante la indicación de la cirugía de EP. Fue posible identificar tanto dudas y miedos sobre el procedimiento y sus resultados, como también sentimientos de alivio, alegría y expectativas positivas ante la cirugía.

Surgieron dudas y miedos en relación al procedimiento en sí (anestesia y recuperación de la EP) y también en relación a sus resultados (ostomías, cambios corporales, miedo a la muerte): "Que yo pienso así en la recuperación, si voy a vencer esa etapa de la recuperación, si voy a vencer aquella anestesia..." (P2, 48 años, separada, cáncer de cuello de útero, 15 meses en tratamiento quimioterapia y radioterapia); “Ah, una piensa muchas cosas, ¿no? ¡Pensamos muchas tonterías! (...) Pensé que no funcionaría, a veces. (...) Pensé que no valía la pena, porque vemos a los vecinos que se van ino?, por los mismos motivos [cáncer]. (...) Entonces pensé: tanto trabajo, así, que válgame Dios, ¿no? ¡Para que después no funcione y morirme igual! (...) Entonces decidi que lo quería hacer para que no avance más [el cáncer]. (...) Si yo lo dejo, pues... ya que tengo miedo que me pase alguna cosa, pues va a ser peor ¿no?" (P1, 37 años, casada, cáncer de cuello de útero, 36 meses en tratamiento - histerectomía, braquiterapia, quimioterapia y radioterapia); "Tenía ganas de irme corriendo a casa. No, no era ni una fuga, yo me sentía con ganas de irme a casa y despedirme... Pensé que iba a entrar en cirugía y no iba a salir. (...) Es que también pensé que aunque saliese de cirugía con bolsa, iba a salir prácticamente sin nada, solo iba a vegetar, no iba a vivir. (...) Pensé que sí, que iba a caminar, pero que la vida no iba a tener gracia. Que no me iba a dar placer ir a un salón de belleza a hacerme las cejas, o comprarme una ropa. Que solo iba a estar ahi por estar, ¿sabe? (...) En casa nos gusta mucho pasear, salir de noche y esas cosas. Entonces todo eso lo pensaba yo, ¿no, doctora? que no voy a tener ningún placer más en la vida, y digo yo que no sé si mi marido va a querer salir más conmigo..." (P4, 53 años, casada, cáncer de cuello de útero, 36 meses en tratamiento - braquiterapia y radioterapia).

Aun así, también se identificaron relatos indicativos de alivio y alegría, por la EP ser comprendida como el último recurso de cura ante el cáncer, así como expectativas positivas ante la cirugía, esto es, de éxito del procedimiento: "Espero que la cirugía sea cuanto antes, ¿no? que no tarde mucho (...) Espero salir de aqui buena (...) yo lo hice todo bien, no fallé nada hasta ahora” (P1); "El doctor me lo explicó así: si no me hiciese la cirugía, a cada minuto yo me iba pudriendo. Él dijo que el olor es insoportable. Que las personas no iban a aguantar estar a mi alrededor, ni los familiares, y que ni yo misma me iba a aguantar. Entonces yo pensé, y entonces él me habló de los dolores, también. Pensé: de qué me va a servir, para qué voy a estar pasando ese dolor, una cosa que antes podría haber evitado ¿no? Por eso pensé: puedo hasta quedarme fea, deformada, pero por lo menos yo no voy a tener dolor. (...) El olor, y la enfermedad creciendo, contando cada minuto ¿no? creciendo y yo sintiendo las personas huyendo de mi ¿no, doctora? Eso me aterrorizó... Y sin posibilidad de cura ¿no? (...) Así, estoy luchando, como el doctor me dijo, que es $40 \%$ de posibilidad. 
Pero yo me voy a agarrar a ese 40\%, que ese 40\% para mí... digamos que es 90\% para mi" (P4); "Yo ya tengo seis hijos, asi que si hay que sacarlo todo, por mi todo bien. (...) Yo ya sabía que si me sacaban el útero, si me sacan lo que ya no vale, pues fuera. (...) Lo que sea bueno para mi puede hacerlo, yo quiero la cura, quiero estar buena, quiero tener un buen tiempo más de vida, criar a mis hijos. Por mí, lo que ustedes hagan por mi salud, iperfecto! (...) Yo me decidi por lo siguiente: ya lo intenté de una forma que fueron las quimios, que era la oportunidad que tenía de curarme con esos tratamientos (...) y ya no existe otra más que la cirugía. Yo ya quería antes la cirugía, pero lo intentamos de la otra forma, pero desgraciadamente no funcionó. Pues a la siguiente. Vamos a hacer la cirugía, sacarlo fuera y a seguir la vida. (...) Yo pensaba yo sola: ¿Y si no me llaman? Yo me muero de ganas de que me llamen para hacer esa cirugía de una vez, para fuera, porque una se pone aprensiva ¿no? (P3, 39 años, viuda, cáncer de cuello de útero, 48 meses en tratamiento - braquiterapia, quimioterapia y radioterapia); "Yo ya tenía la idea que quería hacerme esa cirugía hace mucho tiempo, yo tenía muchas ganas de hacerla (...) No conocía profundamente, pero yo quería hacérmela para poder.. yo sabía que si me hiciese la cirugía me iba a recuperar. Que me iba a salvar. (...) Cuando el doctor me lo dijo, yo me puse muy contenta, porque él me dio mucha esperanza. Dijo que tenía todas las oportunidades del mundo de recuperarme (...) Yo ya tenía esa idea, ya quería antes [operarme]. (...) Porque era la única solución... si no me hago la cirugía ¿qué más voy a hacer? La radio no puedo hacerla más, porque mi cuerpo ya está lleno de radiación ¿no? ya paso del límite. Entonces, la única cosa que me salva ahora es la cirugía. (...) Yo espero que todo salga bien, doctora, espero que sea satisfactoria mi cirugía” (P2).

\section{2) Sentimientos en relación a las ostomías}

En esta categoría fueron considerados todos los fragmentos de entrevistas en los que las participantes expresan sentimientos en relación a las posibles ostomías. Fueron identificados miedo, preocupación y ansiedad ante esta posibilidad, tanto por las reacciones de las otras personas como por el hecho de este cambio ser definitivo y representar la pérdida de una parte del propio cuerpo: "Me quedé asustadísima... me dijeron que iba a tener que colocarme dos bolsitas a la vez. (...) Yo pensé que iba a ser una operacioncita más simple, pero dos bolsas... ¡Madre mía, es duro! (...) Pues da miedo ¿no? (...) Entonces pregunté si la bolsa era definitiva y ellos me dijeron que sí, que era definitiva" (P6, 64 años, casada, cáncer de cuello de útero, 12 meses de diagnóstico, tratamiento ambulatorio con ginecólogo); "Ellas me explicaron que iba a tener que hacerme una cirugía grande, tendría que ponerme una bolsita. Entonces me quedé un poco traumatizada, me dio un pánico, un shock ¿no? (...) Asi que tengo mucha ansiedad, sabe (...) Y ellas me dijeron también que voy a ser la misma mujer, ¿no?, pero así, que no va a cambiar nada. No sé, tengo mucho miedo (...) por mis hijos pequeños, sabe, me da mucha pena que tengan que ver esas cosas colgando de mí" (P1); "Yo voy mucho a la iglesia y dicen que la bolsa de la vejiga es una bolsa grande, tiene una manguerita. Y yo lo he visto ¿sabe?, no me dijeron nada, pero veo a las mujeres usándola. Entonces intento no saber si realmente 
él [médico] va a... (...) Solo pregunto: ¿¿Y los exámenes? ¿Los resultados ya salieron?'. Tanto es así que no le pregunté [al médico] si en ese examen primero que me hice ya veía la vejiga. No tuve coraje de preguntarle, por la bolsita. Vanidad, nada más, ¿no?” (P3); "Yo le dije [al compañero]: 'Vamos a quedarnos solo nosotros, no quiero... o sea, no quiero que la gente intente mirarme'. (...) Por eso doctora, me da miedo hasta de pensarlo, porque yo siempre trabajé en mi vida. Me encanta trabajar. Y entonces, pues sabe, doctora, no puedo imaginarme volver, como lo diría, por la mitad... (...) No puedo imaginarme yo volviendo con una bolsa. (...) Y pienso así: ¿y si voy al baño y automáticamente una de mis amigas abre la puerta? Si... ay, no sé, ¿y si alguien se me aproxima, me toca y la siente?" (P4).

\section{3) Sentimientos en relación a las repercusiones de la EP en el cuerpo y la sexualidad}

Esta tercera categoría temática englobó los fragmentos de entrevista en las que las participantes se referían a sentimientos sobre las posibles alteraciones corporales y de la sexualidad tras el EP. Fueron encontrados tanto relatos de aceptación de esos cambios, como también miedo a la reacción personal y a la reacción del compañero ante los mismos.

Las participantes demostraron una aceptación ante los posibles cambios corporales como consecuencia de la EP y sus inevitables repercusiones en la sexualidad: "No, para mí, normal. Primero porque yo ya me metí en la cabeza que yo no tengo novio, no tengo a nadie más, yo tengo un amigo (...) él entendió la situación, él continúa viniendo a verme, él continúa yendo a ver a mis hijos. (...) Pero ganas de volver a tener otra persona... porque comentan que después de hacerte la cirugía, ya no tienes más ganas de tener relaciones y te quedas diferente, porque ya no tienes aquellos órganos que dan excitación, esas cosas, entonces para mí, ya me meti en la cabeza... (...) Yo hasta le hago bromas: 'No te va a servir de nada venir a querer hacer cosas conmigo, porque voy a ser una mujer 'hueca', no vas a tener ningún placer' (...) Hueca es una forma de hablar. Yo creo que... yo digo, voy a volver a ser virgen, pero virgen que nadie puede tocar. Yo tengo en mente que ellos lo van a sacar y aquello se va a quedar cerrado. (...) Es mi pensamiento, no me explicaron nada y yo procuro dejarles [a los médicos] venir y que me digan, no estar yo todo el tiempo presionando: 'iEh, quiero saber, quiero saber!'. (...) Pienso: ellos van a sacar todo, entonces se quedará así, como si estuviera liso, cosido" (P3); "El sexo no es lo único que existe para ser feliz. Hay tantas otras cosas buenas, importantes de hacer. Y otra cosa, el sexo, en mi cabeza, se ha convertido en una cosa tan desvergonzada, tan banal. No sé si es por la televisión... (...) Lo que tenía que vivir, ya lo vivi'”(P2).

Por otro lado, también se encontraron referencias al miedo de una extrañeza ante los cambios corporales y de la sexualidad, así como de la reacción del compañero a tales cambios: "Quedarse sin vagina y sin la parte interna ¿no? (...) Mira, en el momento... yo pensé no sé qué, es decir: ‘Cómo voy a mirarme al espejo?'. Y ahora esta noche, pensé: ¿y mi marido? Es una situación para él, ¿no? (...) Hace 34 años que estamos casados, pero somos bastantes compañeros, ¿sabe? Bueno, pues ahora esta noche estaba pensando que no habia pensado en él. (...) Pues sí, estaba pensando solo en mí. (...) ¿Cómo me voy a cambiar de ropa delante de una 
persona? Pensé en la lencería: ¿Cómo voy a comprarme una lencería si... si ya no va a hacerme falta? (...) Eso me pasó por la cabeza, pero entonces esta noche me vino la cuestión de él. ¿Un hombre se quedará? Creo que no, así, sin una vida activa ¿no? Además, no hay una reconstrucción, ni nada... (...) No, estoy segura de que él no se va, no. En eso estoy segura que no se va, pero es que ahora todavía puedo pensar, ¿no? Claro que él puede arreglarse con una novia, alguna cosa así, ¿pero y al cabo del tiempo yo voy a aceptar eso? Yo me voy a ver toda fea, toda estropeada, ¿no? Y entonces, ¿lo voy a aceptar? (...) Usted sabe, doctora, que hoy fui a hacer pipí, y el pipí así fue bien largo y yo pensé hasta en eso, quiero decir: hasta el placer del pipi lo voy a perder. Que da una sensación así, un gustito, cuando hacemos pipi ¿no?" (P4).

\section{Discusión}

Destacamos que el 84,4\% $(n=5)$ de las participantes habían recibido un diagnóstico de cáncer de cuello del útero, lo que indica la importancia de más acciones preventivas ante una enfermedad agresiva y silenciosa, que ocasiona un trastorno emocional considerable ${ }^{(14)}$. De hecho, en lo que respecta a los aspectos emocionales, el $50 \%$ de las participantes presentaron ansiedad y el 66,7\% depresión.

En general, reacciones de ajuste tienden a ser frecuentes entre pacientes internos en un hospital general, conforme observado por Botega ${ }^{(18)}$, en estudio realizado en enfermería de un hospital universitario en el interior de São Paulo. En particular, en lo relativo al cáncer, los resultados del presente estudio coinciden con aquellos encontrados por Avelar et al. ${ }^{(10)}$, que aplicaron el HADS a 85 pacientes con cáncer de mama que recibieron indicación para cirugía en la ciudad de Campinas/SP, identificando tasas de $40 \%$ de ansiedad y $27 \%$ de depresión en el periodo preoperatorio.

Por otro lado, los índices encontrados en el presente estudio superan aquellos identificados por Skarstein et al. ${ }^{(31)}$. Ese estudio realizado con 353 pacientes femeninas y 215 masculinos en Noruega, evaluando la calidad de vida y niveles de ansiedad y depresión en presencia de diversos tipos de cáncer, empleando la HADS, identificó un $13 \%$ de pacientes con ansiedad y un $9 \%$ con depresión, tanto en los tratamientos ambulatorios cuanto en las internaciones. Tales diferencias pueden ser causados por los tipos de tratamiento y de cáncer investigados, así como por las condiciones económicas y de salud de cada país. Aun así, es perceptible que los pacientes oncológicos acaban sufriendo con síntomas de ansiedad y/o depresivos en mayor o menor grado.

Los resultados del presente estudio también coinciden con la revisión de la literatura llevada a cabo por Turns ${ }^{(12)}$ sobre aspectos psicosociales de la EP. Conforme esa revisión, las pacientes experimentan ansiedad y depresión en el periodo preoperatorio, principalmente cuando no reciben informaciones adecuadas en relación a la cirugía. Otros estudios empíricos también han indicado que, debido a las repercusiones de la EP, las mujeres tienden a presentar conmoción en su equilibrio emocional y cuadros de insomnio y depresión ${ }^{(24)}$. Aunque en el presente estudio no haya sido investigado el periodo postoperatorio, se percibe una coincidencia en relación a la presencia de ansiedad y depresión encontrada por Rezk et al. ${ }^{(24)}$ en pacientes sometidas a la EP. 
También en un estudio norte-americano con 11 mujeres que realizaron la EP, el $67 \%$ de las pacientes presentaron tristeza y depresión ${ }^{(10)}$. Y fueron evidenciados culpa y pensamientos de sentirse una carga para los familiares. Siendo así, es perceptible que los resultados del presente estudio coinciden con aquellos ya identificados en estudios anteriores realizados en otros contextos socioculturales.

Además, los resultados cualitativos coinciden con los datos cuantitativos, pues los sentimientos y percepciones que emergieron en los relatos de las participantes están relacionados a la ansiedad y depresión, demostrando la vivencia de una trayectoria de sufrimiento desde el diagnóstico del cáncer.

Aun considerando la EP como el último recurso de cura, las repercusiones físicas y psicosociales previstas a partir de este procedimiento quirúrgico podrían predisponer a la depresión. Así, los altos índices de ansiedad y depresión identificados en las pacientes podrían estar asociados a la indicación de la EP, considerando que estas mujeres, en alguna medida, preveían la mutilación de su cuerpo y la necesidad de ostomías. En relación a este aspecto, cabe destacar que esas pacientes habían recibido la orientación para la EP en un período próximo a la colecta de datos y posiblemente aun se encontraban bajo el impacto emocional de esta indicación médica. De este modo, estos datos corroboran la literatura, evidenciando la experiencia de episodios reactivos, como ansiedad y depresión, entre las pacientes indicadas para la $\mathrm{EP}^{(10,12,24)}$.

Por otro lado, estas mujeres ya se encontraban en tratamiento oncológico por un tiempo y demostraban temer su nueva condición física, aun más ante el fracaso de los tratamientos realizados hasta entonces. En este sentido, no se puede afirmar que la depresión y la ansiedad presentadas por las pacientes sean consecuencia únicamente de esta indicación quirúrgica. El cáncer es una enfermedad que puede causar dolor, angustia, sufrimiento y que genera muchos cambios en la imagen corporal, por tanto, aquellas pacientes que ya vivieron otras cirugías podrían presentar estrés postraumático, altos niveles de ansiedad y depresión, o incluso alteración de la percepción del cáncer y de sí mismas ${ }^{(32)}$.

El tratamiento, además de dolorido y desgastante físicamente, también lo es emocionalmente, teniendo en cuenta la necesidad de revisión constante y el miedo a la recidiva ${ }^{(10,21,23,33,34)}$. De esta forma, este contexto puede explicar los resultados del presente estudio, más allá de la indicación de la EP. Es factible pensar que esa indicación haya sido un factor estresor adicional para estas mujeres, generando ansiedad por el hecho de la cirugía ser algo desconocido y mutilante, pero también por ser la única posibilidad de cura aun existente. De esta forma, la indicación de EP podría hacer surgir síntomas depresivos ante el luto por el fracaso del tratamiento realizado hasta entonces y por la previsión de pérdida de partes del cuerpo representadas por las ostomías.

Considerando los resultados de forma conjunta, fue posible percibir la intensidad de las repercusiones emocionales de este tipo de cáncer y de la indicación de la EP para las pacientes. Como destacado, el miedo y la preocupación con el uso de bolsas colectoras generaron incomodidad y ansiedad, lo que coincide con los resultados de Dabirian et al. ${ }^{(35)}$. A través de un estudio cualitativo realizado en Irán con 14 pacientes (14 a 57 años) que usaban bolsas de colostomía, los autores concluyeron que muchas de las dificultades relatadas por los pacientes ocurrieron en un periodo inicial tras la cirugía, tales como los cambios en la imagen corporal, ansiedad sobre los escapes de heces en las bolsas, el olor, los ruidos intestinales y el empobrecimiento de la libido. Con el paso del tiempo, observaron una buena 
capacidad de adaptación, aprendizaje del uso y manejo de la ostomía. Tales aspectos no emergieron en el presente estudio, probablemente por el momento de realización de la entrevista (preoperatorio).

Otro aspecto destacable en este estudio es el miedo de las pacientes a la reacción y los prejuicios de las demás personas ante la presencia de ostomías, más que su propia reacción y la necesidad de adaptación física y psíquica. En estudio realizado también en el sur de Brasil con mujeres con cáncer ginecológico sometidas a EP, se destacaron, entre otros aspectos, la inseguridad sobre la imagen corporal y la disminución de auto-estima relacionada a las ostomías, con énfasis al miedo de abandono por el compañero ${ }^{(36)}$. En esa misma dirección, estudio realizado en Dinamarca con 15 personas con ostomía permanente, también identificó miedo de los prejuicios y preocupación ante la revelación de la ostomía a otras personas. Debido a esto, un comportamiento de aislamiento social autoimpuesto puede ser utilizado como estrategia por los pacientes para evitar esta revelación ${ }^{(37)}$.

Otro tema que destacó en los relatos de las participantes fueron las eventuales repercusiones de la EP sobre la sexualidad. Según Hawighrst-Knapstein et al. ${ }^{(21)}$, cambios en la imagen corporal y en la sexualidad pasaron a ser foco de sufrimiento e inseguridad para pacientes en Alemania. También Brown e Randle ${ }^{(38)}$, en revisión de literatura sobre el uso de bolsas de colostomía, destacaron la imagen corporal, la sexualidad y las preocupaciones sexuales como temas estudiados en esta área. Por tanto, los datos del presente estudio corroboran parcialmente la literatura, ya que fue encontrada una mayor preocupación de las participantes sobre la aceptación de su nuevo cuerpo por el compañero, hijos y demás personas con las que se relacionaban, y no tanto por ellas mismas.

Aunque la necesidad de cambio en la imagen corporal haya sido prevista por las participantes, también fueron encontrados relatos de ausencia de preocupación ante el aspecto sexual. Este dato contradice la literatura, ya que estudios realizados con ese público señalaron la sexualidad como un aspecto bastante perjudicado tras la $\mathrm{EP}^{(10,12,23,24,36)}$. Es plausible pensar que este hallazgo se deba al momento de colecta de los datos (previo al procedimiento), del tiempo de tratamiento de las pacientes (media de 2 años y 4 meses) y/o del sufrimiento ya enfrentado en ese proceso, que incluye, entre otros síntomas, sangramientos vaginales y dolores, problemas en la vida sexual ya vigentes en el momento de la indicación de cirugía.

Desde esta perspectiva, de forma semejante a estudios internacionales ${ }^{(13)}$, en el presente estudio las pacientes destacaron la importancia de enfrentar la EP y adaptarse a los cambios físicos con la intención de vivir. De este modo, se percibe claramente un deseo de curación, estando las pacientes dispuestas a abdicar del propio cuerpo y de su sexualidad en pro de su propia vida.

Es posible suponer que esta motivación por vivir y curarse haya sido responsable de otra diferencia encontrada entre los hallazgos del presente estudio y aquellos de Wrigth et al. ${ }^{(13)}$. En el presente estudio, las pacientes informaron una expectativa positiva en relación a la EP, refiriendo alivio y alegría por tener una nueva oportunidad de tratamiento del cáncer. Este elemento no fue verificado por los autores canadienses; las participantes parecieron decepcionadas ante las limitadas opciones de tratamiento de la enfermedad, aunque también refiriesen la importancia de creer en las posibilidades aun existentes.

El miedo es una respuesta emocional a una amenaza inminente real o percibida, mientras que la ansiedad está relacionada a una amenaza futura ${ }^{(25)}$. Así, miedos y 
dudas de las participantes sobre el procedimiento y sus resultados, encontrados en el presente estudio, parecieron adecuados ante una cirugía de gran envergadura como la EP. En este sentido, 103 pacientes austriacos con cáncer, entrevistados por Hollaus et al ${ }^{(38)}$ antes de una cirugía, también refirieron miedo de la repercusión del procedimiento en su Calidad de Vida. De esta forma, las reacciones emocionales encontradas en el presente estudio parecen relacionadas con el momento vivido por las pacientes, ya que Wright et al. ${ }^{(13)}$ verificaron que aproximadamente el 75\% de las mujeres sometidas a la EP sufren efectos colaterales físicos y enfrentan un largo periodo de recuperación, presentando problemas psicológicos, incluso en el postoperatorio.

\section{Conclusiones}

El presente estudio, por la delineación empleada, permitió la integración de datos cualitativos y cuantitativos para la comprensión de los aspectos psicológicos de mujeres con cáncer indicadas para la cirugía de EP. Ante lo expuesto, se verifica la necesidad de nuevas investigaciones sobre el tema, para que se pueda conocer la vivencia de esas mujeres ante los cambios previstos antes de la EP. Específicamente, estudios longitudinales, con el objetivo de acompañar el enfrentamiento y la reinserción de estas pacientes en el contexto en que viven tras la cirugía, podrían esclarecer la diferencia encontrada entre los hallazgos del presente estudio y la literatura, en lo relativo al impacto de la EP sobre la sexualidad. Además, podrían ampliar el espectro de variables emocionales investigadas, incluyendo el análisis de otras comorbilidades psiquiátricas.

Además de esto, los hallazgos del presente estudio destacan la necesidad de un acompañamiento psicológico prequirúrgico para mujeres indicadas a un procedimiento altamente especializado e invasivo como la EP, contribuyendo a amenizar sus dolores en esta trayectoria repleta de desafíos rumbo a la supervivencia.

\section{Agradecimientos}

Dr. José Pio Furtado, cirujano oncológico del Hospital Santa Rita (ISCMPA), por el apoyo al estudio y auxilio en el contacto inicial con las participantes. Estudiante de Grado Flávia Santos da Silva, del Curso de Psicología de la UFCSPA, por el auxilio en la diagramación de este artículo. Al CNPq (Conselho Nacional de Desenvolvimento Científico e Tecnológico) por la Beca de Produtividad en Investigación de Daniela Centenaro Levandowski”.

\section{Referencias bibliográficas}

1. INCA - Instituto Nacional do Câncer. Estimativas 2016: Incidência de Câncer no Brasil. Rio de Janeiro: Inca. [acesso em 02 ago 2016] Disponível em: www.inca.gov.br

2. Franco VF. Exenteração pélvica no câncer ginecológico. In: Santos CER, Mello EL, editors. Manual de Cirurgia Oncológica. 2a .ed. São Paulo: Tecmedd, 2008; p. 593-603. 
3. Zardo GP, Farah FP, Mendes FG, Santos Franco CAG, Molina GVM, Melo GN, et al. Vacina como agente do HPV. Ciênc saúde coletiva 2014;19:3799-808.

4. Peretto M, Drehmer LBR, Bello HMR. O não comparecimento ao exame preventivo do câncer de colo de uterino: razões declaradas e sentimentos envolvidos. Cogitare enf 2012;17:29-36.

5. Diver JE, Rauh-Hain JA, Carmen MG. Total pelvic exenteration for gynecologic malignancies. Int J Surg Oncol Article ID 693535, 9 pages, 2012. doi:10.1155/2012/693535

6. Brunschwig A, Daniel W. Observations on the urinary tract four to seven years after total pelvic exenteration and wet colostomy. Ann Surg 1955;142:729-38.

7. Silva JH. Exenteração pélvica e tratamento do câncer do reto. Arq Gastroenterol 2007;44:283. doi: 10.1590/S0004-28032007000400002

8. Costa SRP, Teixeira ACP, Lupinacci RA. A exenteração pélvica para o câncer de reto: avaliação dos fatores prognósticos de sobrevida em 27 pacientes operados. Rev Bras Colo-proctol 2008;1:7-18. doi:10.1590/S0101-98802008000100001

9. Garza J, Wilson S, Behbakht K. Total pelvic exenteration with a split-thickness skin graft neovagina, continent orthotopic neobladder and rectal reanastomosis, resulting in no external ostomies and adequate sexual function. Gynecol Oncol 2009;115:312-3. doi: 10.1016/j.ygyno.2009.07.002

10. Carter J, Chi D, Abu-Rustum N, Brown LC, Mccreath W, Barakat R. Brief report: Total pelvic exenteration - A retrospective clinical needs assessment. Psychooncology 2004;13: 125-31. doi:10.1002/pon.766

11. Guimarães GC, Baiocchi G, Ferreira FO, Kumagai LY, Fallopa CC, Aguiar S, et al. Palliative pelvic exenteration for patients with gynecological malignancies. Arch Gynecol Obstet 2011; 283:1107-12. doi:10.1007/s00404-010-1544-8

12. Turns D. Psychosocial issues: Pelvic exenterative surgery. Int J Surg Oncol 2001;76: 224-36. doi:10.1002/jso.1036

13. Wright F, Crooks D, Fitch M, Hollenberg E, Maier BA, Last LD, et al. Qualitative assessment of patient experiences related to extended pelvic resection for rectal cancer. J Surg Oncol 2006;93:92-9. doi: 10.1002/jso.20382

14. Castro EK, Aretz M, Lawrenz P, Romeiro FB, Haas SA. Illness perceptions in Brazilian women with cervical cancer, women with precursory lesions and healthy women. Psicooncología 2013;10:417-23. doi:10.5209/rev_PSIC.2013.v10.n2-3.43459

15. Schávelzon, J. Sintomatologia y tratamiento. In: Psique, cancerologia, psicologia, psicoterapia e psicofarmacos. Buenos Aires: Científica Interamericana, 1992; p. 64-119.

16. Fernandes WC, Kimura M. Calidad de vida relacionada a la salud de mujeres con cancer de cuello uterino. Rev Latino-Am Enfermagem 2010;18:65-72.

17. Estapé T. Disfunciones sexuales em pacientes de câncer. In: Dias MR, Dura E, editors. Territórios da Psicologia Oncológica. Lisboa: Climepsi, 2001; p. 481-92.

18. Botega NJ. Reação à doença e à hospitalização. Prática Psiquiátrica no Hospital Geral: Interconsulta e Emergência . 3th ed. Porto Alegre: Artmed, 2012; p. 46-61.

19. Neme CMB, Bredariolli RNB. Mulheres com câncer de mama, de útero e de ovários: Estudos clínicos de casos. In: Neme CMB, editor. Psico-Oncologia: Caminhos e Perspectivas. São Paulo: Summus, 2010. p. 99-147.

20. Talgham C. Una mirada psicoanalítica acerca del câncer ginecológico. In: Alkolombre P., editor. Travesías del Cuerpo Feminino. Buenos Aires: Letra Viva, 2011. p. 233-62. 
21. Hawighrst-Knapstein S, Fusshoeller C, Franz C, Trautmann MS, Pilch H, Schoenefuss $\mathrm{G}$, et al. The impact of treatment for genital cancer on quality of life and body image: Results of a prospective longitudinal 10-year study. Gynecol Oncol 2004;94:398-403. doi: 10.1016/j.ygyno.2004.04.025

22. Ross EJ, Graeff A, Eijkeren MA, Boon TA, Heintz APM. Quality of life after pelvis exenteration. Gynecol Oncol 2004;93:610-4. foi: 10.1016/j.ygyno.2004.03.008

23. Pusic AL, Mehrara BJ. Vaginal reconstruction: An algorithm approach to defect classification and flap reconstruction. J Surg Oncol 2006;94:515-21. doi: 10.1002/ jso.20489

24. Rezk YA, Hurley KE, Carter J, Dao F, Bochner BH, Aubey JJ, Caceres A, et al. A prospective study of quality of life in patients undergoing pelvic exenteration: Interim results. Gynecol Oncol 2013;128:191-7. doi: 10.1016/j.ygyno.2012.09.030

25. American Psychiatric Association. Manual Diagnóstico e Estatístico de Transtornos Mentais - DSM-5. 5th ed. Porto Alegre: Artmed, 2014.

26. Creswell JW. Projeto de Pesquisa: Métodos Qualitativo, Quantitativo e Misto. 3th ed. Porto Alegre: Artmed, 2010.

27. Marcolino J, Mathias L, Piccinini Filho L, Guaratini A, Suzuki F, Alli L. Escala Hospitalar de Ansiedade e Depressão: Estudo da validade de critério e da confiabilidade com pacientes no pré-operatório. Rev Bras Anestesiol 2007;57:52-62. doi: 10.1590/ S0034-7092007000100006

28. Botega NJ, Bio MR, Zomignani MA, Garcia Junuir C, Pereira WAB. Transtornos do humor em enfermaria de clínica médica e validação de escala de medida (HAD) de ansiedade e depressão. Rev Saúde Pública 1995;29:355-63. doi:10.1590/S003489101995000500004

29. Laville C, Dionne J. A Construção do Saber: Manual de Metodologia da Pesquisa em Ciências Humanas. Porto Alegre: Artmed, 1999.

30. Avelar AMA, Derchain SFM, Camargo CPP, Lourenço LS de, Sarian LOZ, Yoshida A. Qualidade de vida, ansiedade e depressão em mulheres com câncer de mama antes e após a cirurgia. Rev. ciênc. méd., (Campinas) 2006;15:11-20.

31. Skarstein J, Aass N, Fossa SD, Skovlund E, Dahl AA. Anxiety and depression in cancer patients: relation between the Hospital Anxiety and Depression Scale and the European Organization for Research and Treatment of Cancer Core Quality of Life Questionnaire. J Psychosom Res 2000;49:27-34.doi: 10.1016/S0022-3999(00)00080-5

32. Castro EK, Romeiro FB, Lima NB, Lawrenz P, Haas S. Percepção da doença, indicadores de ansiedade e depressão em mulheres com câncer. Psicol saúde doenças 2015;16:359-72. doi: 10.15309/15psd160307

33. Black EK, White CA. Fear of recurrence, sense of coherence and posttraumatic stress disorder in haematological cancer survivos. Psychooncology 2005;14:510-5. doi: 10.2147/PPA.S14508

34. Lee-Jones C, Humphris G, Dixon R, Hatcher MB. Fear of cancer recurrence-a literature review and proposed cognitive formulation to explain exacerbation of recurrence fears. Psychooncology 1997;6:95-105. doi: 10.1002/(SICI)1099-1611(199706)6:2<95::AIDPON250>3.0.CO;2-B

35. Dabirian A, Yaghmaei F, Rassouli M, Tafreshi MZ. Quality of life ostomy patients: a qualitative study. Patient Prefer Adherence 2011;5: 1-5. doi: 10.2147/PPA.S14508

36. Huff R, Castro EK. Repercussões emocionais do câncer ginecológico e exenteração pélvica. Rev Psicol Saúde 2011;3:33-42. 
37. Danielsen AK, Soerensen EE, Burchaeth K, Rosenberg J. Learning to life with a permanent intestinal ostomy: impact on everyday life and educational needs. J Wound Ostomy Continence Nurs 2013;40:407-12. doi10.1097/WON.0b013e3182987e0e

38. Brown H, Randle J. Living with a stoma: a review of the literature. J Clin Nurs 2005;14:74-81. doi:10.1111/j.1365-2702.2004.00945.x 